\title{
Multilinguales
}

\section{Aventure de la lexie « mot » dans Moi, Laminaire d'Aimé Césaire : rendement stylistique, signifiance et perspectives}

Adventure of the lexical item "word" in Moi, Laminaire of Aimé Césaire, Stylistic performance, significance and perspectives.

$$
\text { لإيمي سيزير, المردودية الأسلوبية, موا لامينار مغامرة اللكسيم "كلمة" في }
$$

\section{Sylvanius Bobo Rostand}

\section{CpenEdition \\ Journals}

Édition électronique

URL : http://journals.openedition.org/multilinguales/1100

DOI : $10.4000 /$ multilinguales. 1100

ISSN : 2335-1853

Éditeur

Université Abderrahmane Mira - Bejaia

\section{Référence électronique}

Sylvanius Bobo Rostand, « Aventure de la lexie « mot » dans Moi, Laminaire d'Aimé Césaire : rendement stylistique, signifiance et perspectives », Multilinguales [En ligne], 9 | 2018, mis en ligne le 01 juin 2018, consulté le 10 décembre 2020. URL : http://journals.openedition.org/multilinguales/1100; DOI : https://doi.org/10.4000/multilinguales. 1100

Ce document a été généré automatiquement le 10 décembre 2020.

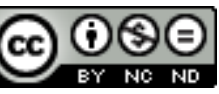

Multilinguales est mise à disposition selon les termes de la Licence Creative Commons Attribution Pas d'Utilisation Commerciale - Pas de Modification 4.0 International 


\title{
Aventure de la lexie « mot » dans Moi, Laminaire d'Aimé Césaire : rendement stylistique, signifiance et perspectives
}

\author{
Adventure of the lexical item "word" in Moi, Laminaire of Aimé Césaire, \\ Stylistic performance, significance and perspectives. \\ لإيمي سيزير, المردودية الأسلوبية, موا لامينار مغامرة اللكسيم "كلمة" في \\ دلالة وتوقعات
}

\section{Sylvanius Bobo Rostand}

Aventure, sommairement, renvoie à une action, mieux, un mouvement, un voyage singulier qui comporte une dose d'imprévisibilité, d'inconnu et d'extraordinaire. Quant au mot, il s'appréhende comme la projection sur la chaîne parlée du signe linguistique. Il est défini comme " une masse sonore dont l'émission et la réception suggère à la conscience une représentation sensible ou une notion spéculative » (Mazaleyrat et Molinié, 1989 : 229). Il a pour équivalent la lexie, terme que Mazaleyrat et Molinié jugent beaucoup plus commode car "l'identification, et partant l'existence [du mot] n'est pas évidente » (ibid.). Ils insisteront sur le fait que la lexie est « la plus petite unité séparable et commutable dans le discours...Une lexie constitue un signe, dont les termes d'analyse sont le signifiant et le signifié » (1989 : 196). La lexie, à l'image du signe linguistique, est composée d'un signifiant et d'un signifié, ce dernier se présentant comme la somme de composantes dénotatives et connotatives. La question de l'aventure du mot peut être abordée selon la double perspective diachronique et synchronique, dichotomie chère à Saussure (1916: 127-134), en matière d'études linguistiques. La perspective diachronique, décrirait la trajectoire du mot, ses différentes péripéties, depuis son apparition dans la langue jusqu'au moment où l'on aborde son étude. En effet, au cours de leur «vie ", les mots subissent des transformations aussi bien morphologiques que sémantiques. Par exemple, du point de vue sémantique, les mots subissent tantôt un affaiblissement, tantôt un renforcement ou une mutation de sens ou tout simplement « meurent ", c'est- 
à-dire sortent de l'usage. Cette aventure, qui est l'histoire particulière d'une lexie, intéresse prioritairement les lexicologues. S'agissant de la perspective synchronique, laquelle préoccupe la stylistique, l'aventure du mot s'intéresse non plus à la trajectoire chronologique du mot, mais plutôt de ses « avatars" consécutifs à la tension entre son sens de base et les différentes valeurs que son actualisation dans le discours lui impose. Elle s'apprécie dans le hic et nunc du discours littéraire, en l'occurrence. Étudier l'aventure de la lexie « mot » dans Moi, laminaire de Césaire revient, alors, à analyser les différentes valeurs de cette lexie, le rendement stylistique et la signifiance (comme production de sens ou d'une sémantique spécifique) consécutifs. Nous préciserons, préalablement et de manière plus explicite, ce que l'on doit entendre par aventure $d u$ mot. L'application de cette méthode d'analyse des textes littéraires, initiée par Bernard Zadi Zaourou, nous donnera la latitude, in fine, de la soumettre à critique pour en jauger l'efficience avant de souligner les perspectives éventuelles qu'elle ouvre à la critique des textes littéraires.

\section{Notes brèves sur « l'aventure du mot »}

2 Roland Barthes, Roman Jakobson et Aimé Césaire sont les trois principaux auteurs qui ont inspiré cette approche des textes littéraires. Zadi Zaourou les cite ou commente leurs travaux dans ce qui a d'abord été un séminaire (maîtrise de lettres modernes) animé par ce dernier à l'université d'Abidjan avant d'être publié en 1994 dans la revue Cuvres et critiques.

3 Dans le Degré zéro de l'écriture (1972: 35-42), Barthes prend pour point de départ le distingo qu'il opère entre prose (discours minimum, véhicule le plus économique de la pensée) et poésie moderne. Entre ces deux pôles, il intercale la poésie classique qu'il présente comme une "variation ornementale de la prose", ce que sa formule célèbre suivante exprime clairement : Poésie (acception classique) $=$ prose $+a+b+c$. A, b et c étant des « attributs particuliers du langage, inutiles mais décoratifs, tel le mètre, la rime, le rituel des images " (Barthes, 1972: 35). Ainsi, dans la prose comme dans la poésie classique, Barthes estime que la précellence revient à l'horizontalité (axe syntagmatique). En effet, souligne-t-il :

L'économie du langage classique (Prose et Poésie) est relationnelle, c'est-à-dire que les mots y sont abstraits le plus possible au profit des rapports. Aucun mot n'y est dense par lui-même, il est à peine le signe d'une chose, il est bien plus la voie d'une liaison. Loin de plonger dans une réalité intérieure consubstantielle à son dessin, il s'étend, aussitôt proféré, vers d'autres mots, de façon à former une chaine superficielle d'intentions (Barthes, $1972: 37$ ).

En clair, le rapport prime sur la quintessence du mot, sa substance. A contrario, la poésie moderne, précisément depuis Arthur Rimbaud, se veut une apologie de la verticalité (axe paradigmatique). A la racine de cette inversion des propriétés naturelles du langage, de l'abolition de l'horizontalité, il y a le «temps poétique » :

La parole est alors le temps épais d'une gestation plus spirituelle, dans laquelle la pensée est préparée, installée peu à peu par le hasard des mots. Cette chance verbale, d'où va tomber le fruit mûr d'une signification, suppose un temps poétique qui n'est plus celui d'une fabrication, mais celui d'une aventure possible, La rencontre d'un signe et d'une intention (Roland Barthes, 1972 : 36).

5 Roman Jakobson, pour sa part, ne mentionne pas spécifiquement cette aventure, mais la définition qu'il donne de sa «fonction poétique » établit, elle aussi, le primat de la 
verticalité, laquelle se caractérise par ce qu'il nomme la "projection de l'axe des paradigmes sur l'axe des syntagmes». Cette irruption de l'axe de la sélection sur l'axe des combinaisons concourt à la neutralisation de ce dernier, mieux à sa négation tant l'axe syntagmatique est mis sens dessus-dessous. Au final, on n'a plus deux axes distincts mais confondus. Chez lui, comme chez Barthes, le mot est « rebelle » parce qu'après sa sélection par le locuteur sur l'axe des paradigmes, il refuse de se plier à la syntaxe nécessaire à la formation de phrases ou d'énoncés. La " fonction poétique » se reconnait, alors, par l'accent mis sur le mot pour lui-même et non plus sur sa vocation naturelle à s'associer à d'autres mots pour former la chaine parlée.

6 Après les poéticiens, le poète Césair ${ }^{1}$, pour qui la « connaissance scientifique » diffère de la « connaissance poétique » en ceci que la première, à rebours de la seconde, est « sommaire, pauvre et famélique » pour la simple raison qu'elle reste à la surface des choses et que l'essence, le «quid proprium» lui échappe. C'est pourquoi, ajoute-il, " la connaissance scientifique nombre, mesure, classe et tue ». A cette connaissance qui "dépersonnalise, désindividualise " fait pièce la connaissance poétique, "rassasiante " par excellence. Au cœur de celle-ci, liberté et imagination règnent en maitresses absolues.

7 Le mot est le véhicule de cette aventure qui est aussi celle du poète. C'est par elle qu'il accède à l'essence des choses, au noumen. Le rapport du poète au mot devient on ne peut plus clair. Homme libre, amoureux de la liberté, il ne saurait soumettre le mot à sa stricte "ustensilité " pour paraphraser Sartre (voir Qu'est-ce que la littérature?), à l'expression la plus sèche possible d'une pensée; ce qui ne ferait que le confiner dans une simple fonction de véhicule de l'information. Loin s'en faut, « le poète vrai souhaite abandonner le mot à ses libres associations, sûr que c'est en définitive l'abandonner à la volonté de l'univers » (Césaire, 1973 : 100). Et Césaire de citer l'humour, l'image, le mythe comme les moyens par lesquels le mot permet au poète d'arriver à ses fins.

8 Césaire, comme Barthes et Jakobson, met l'accent sur la liberté. Il ressort de leurs analyses que le poète, amant de la liberté, n'a d'autre souci que de libérer le mot, ce qui l'engage dans une sorte d'élan sublimatoire de son matériau de base: le mot. Ces différents auteurs, et pas eux exclusivement, s'ils avaient pressenti l'aventure, c'est à Zadi Zaourou que revient le mérite de l'avoir décrite systématiquement en vue d'en faire une méthode d'analyse des textes littéraires. Qu'entend-il par aventure du mot? En quoi consiste-elle précisément? Quelles sont les principales étapes ou degrés de cet « élan vertical»?

9 La première partie de son article est consacrée à la détermination de la place du mot dans la poétique de Césaire. Il y note que le mot (qui donne son nom à de nombreux poèmes ou en constitue la thématique principale) est une "réalité concrète ", tour à tour " arme de combat ", "monstre sacré ». Il décrit la relation entre le mot et la poésie en des termes imagés : «le mot est à la poésie en tant qu'absolu du beau, ce qu'est le rayon solaire au soleil : il faut l'intégrer, l'habiter comme une fusée pour accéder à la source de l'irradiation" (Zadi Zaourou, 1994 : 35).

10 La seconde partie, aborde l'analyse du mot du point de vue linguistique, à juste titre d'ailleurs; le mot étant d'abord et avant tout une réalité de la langue. En le définissant comme la "projection du signe linguistique ", sa "matérialisation», Zadi Zaourou s'inscrit dans le droit fil du saussurianisme. En tant que tel, le mot relève de la parole et comme le signe qu'il reflète, est composé d'un signifiant et d'un signifié, le mot aussi est une réalité biface constituée d'une forme (sonore ou graphique) et d'un contenu 
sémantique (l'image conceptuelle). Ce postulat lui permet d'établir la différence entre le sens et la signification qui se ramène à la formule suivante :

Sens : Sa+Sé< langue

Signification : Sa + Sé + référent < parole. (Ibid.)

11 Expressis verbis, la signification se joue dans ce que Fromilaghe et Sancier appellent le « triangle sémiotique » (Fromilaghe et. Sancier, 1991 : 2) dont les trois pôles sont le signifié, le signifiant (réalités linguistiques) et le référent (réalité extralinguistique). A contrario, la langue est le champ où se lit le sens lequel est alors latent, potentiel comme tout ce qui demeure sur l'axe des paradigmes. Comme il le précise lui-même :

Contrairement au sens qui est statisme, la signification est dynamique. C'est un processus par lequel se réalise justement le sens. Elle seule permet le passage du plan de la langue au plan de la parole, la mutation du signe en mot, par un saut brusque et qualitatif, le mouvement et l'organisation du flux lexical de l'axe paradigmatique vers l'axe syntagmatique. Et c'est pourquoi la signification s'observe toujours en contexte et en rapport avec les référents. (Zadi Zaourou, $1994: 42)$.

12 L'aventure dont il est ici question ne se réalise ni au niveau du sens (qui est enfermement, fixisme, stagnation) ni au niveau de la signification (même si, déjà, à ce niveau s'entame la négation de la rigidité du sens), mais bien plus au niveau de la valeur, au sens saussurien du terme. Cette notion découle de la conception de la langue comme système (structure pour ses disciples) (Benveniste, 1966: 91-98). Ainsi, «les éléments linguistiques n'ont aucune réalité indépendamment de leur relation au tout " (Ducrot et Todorov, 1966 : 32). Dans cette perspective, pour saisir le signe, il faut entrer dans le jeu global de la langue. Ceci est valable pour le mot dans la parole. Portée à son acmé, la valeur se transmute en littérarité.

13 A l'instar de Barthes qui opposait la prose/poésie classique à la poésie moderne, Zadi Zaourou va mettre en parallèle le discours utilitaire et le discours littéraire, ce dernier étant présenté comme le lieu par excellence de l'aventure du mot :

toute œuvre poétique véritable est subversion et, toujours par rapport au despotisme de la langue en tant que système normalisé, hymne à la liberté. Le mot, arme du poète, ne peut évidemment que servir la cause de cette quête libertaire qui commence, bien évidemment, par sa propre libération qui est un processus tumultueux, admettant des étapes que nous avons baptisé "Aventure du mot" (Zadi Zaourou, ibid.)

14 Cette aventure se présente comme un mouvement ascendant, gradué, qui pourrait être représenté sous forme d'échelle dont les principales marches (degrés d'expressivité) sont :

- le "degré zéro"2 : il renvoie au code utilitaire qui somme le mot de dire les choses telles qu'elles sont, sans apprêts ni fioriture. Primauté, à ce stade, à la dénotation.

- $1^{\text {er }}$ degré : il renvoie aux connotations en général. C'est le lieu par excellence des sens seconds. Ce champ est extrêmement ouvert quand le mot est pris isolément, il se restreint quand le mot s'intègre à une structure phrastique ou discursive. A ce stade de l'aventure, il y a frémissement, vrombissement, avant que ne s'engage cet élan icarien, à la limite.

- 2è degré: le niveau figural fait partie du vaste champ des connotations, certes, mais il est isolé pour des besoins pratiques. A ce stade, le mot subit des transferts de sens (tropes et autres figures connexes) ou tout simplement le locuteur joue sur le matériel sonore. Le jeu sur le signifiant et le signifié est particulièrement productif en matière figurale. 
- 3è degré : Comme le niveau figural, lui aussi relève du discours connoté, à la seule différence que les symboles anagogiques au lieu de jouer sur les similitudes ou les rapports de contigüité, introduisent dans le discours des légendes, des mythes.

L'aventure, dont il est ici question, est rébellion contre le code linguistique. Sur le plan phrastique, elle pourrait consister en la violation des règles syntaxiques comme l'ellipse des chainons qui assurent la liaison entre les lexies (accumulations de lexies sans lien syntaxique). Cette agrammaticalité peut se doubler ou non d'une rupture de l'acceptabilité sémantique de la phrase. Dans l'un ou l'autre cas, il s'agit de la précellence de la verticalité (axe paradigmatique) sur l'horizontalité (axe syntagmatique). Mais, c'est à partir du mot que cette aventure s'apprécie le mieux. Elle nait de la tension entre signifiant et signifié, de la pression à laquelle le poète ou le littérateur soumet le mot pour l'exiler le plus loin possible de son sens de base. Elle nous apparait comme un acte volontaire ou non, mais expressif.

Étudier l'aventure du mot, en somme, reviendrait à repérer et à analyser tous les mots employés hors de leur sens de base, à déterminer le degré d'aventure de chaque lexie avant d'évaluer le degré de littérarité du texte ou son épaisseur littéraire, à partir de leur abondance ou surabondance. Dans le cas d'espèce, notre étude s'emploiera à l'examen des avatars d'une lexie unique (mot) à partir de ses différentes occurrences, à en souligner l'expressivité avant de proposer à partir de leur mise en corrélation une hypothèse interprétative des poèmes concernés dans Moi, laminaire d'Aimé Césaire.

\section{Valeur expressive et rendement sémantique de la lexie «mot»}

Dans le poème Mot macumba, la lexie «mot " est employée autant de fois qu'il y a de versets, c'est-à-dire onze itérations au total, le titre excepté. La prééminence de cette lexie est soulignée par ses nombreux retours et de manière plus évidente encore par le fait que chaque verset est construit autour de celle-ci. Mot apparait comme le point nodal de chaque verset. Ces versets s'appellent les uns les autres par la répétition du premier membre, au point de former un schème anaphorique : schème $1:$ «le mot est... "; schème $2:$ : il y a des mots... ».

18 Aucune occurrence n'est exprimée au degré zéro. Le verset 5 aurait pu s'en rapprocher n'eût été le verbal "dessiner » dont il est le complément d'objet et qui relève du paradigme de l'art. Ce même schème anaphorique se retrouve également dans le poème «Sentiments et ressentiments des mots » (23-24) : «le mot oiseau-tonnerre » (24).

19 L'aventure de la lexie se situe surtout au niveau figural. Ce sont presqu'exclusivement des métaphores adjectivales, précisément des substantifs employés comme adjectifs. Au cours de cette aventure, mot visite plusieurs isotopies: le minéral (mot frais, mots d'ombre avec des réveils en colère d'étincelles), le végétal (bâton de nage) et l'animal (iguanes, phasmes, dauphin). Les trois règnes sont investis. A ces réalités profanes, Césaire mêle le sacré au travers de lexies comme macumba, shango et saints. Les deux premiers ressortissent à l'isotopie du vaudou alors que saint relève du christianisme. Cette mixité (règne animal-végétal-minéral) préfigure une certaine "fraternité de tous les étants ». Le poète mêle aussi le sacré et le profane, ce qui pourrait laisser croire à un certain éloge du syncrétisme religieux si caractéristique des milieux nègres des Amériques. Mais, la piste la plus plausible est révélée par le titre thématique du poème : 
Mot macumba. Macumba est un culte proche du vaudou pratiquée dans certaines régions du Brésil, il revendique à la fois des racines africaines (yoruba, éwé, fon, bantou) et amérindiennes. Ses adeptes avaient pour coutume de déguiser leurs divinités sous les noms et les attributs des saints pour échapper à la persécution des Portugais : une des formes embryonnaires du marronnage ${ }^{3}$, thème si cher à Césaire.

D'une manière générale, le mot est pour Césaire une réalité dialectique, tout à la fois doux, fragile (mot couresse) et violent (mots d'ombre avec des réveils en colère d'étincelles) ; profane et sacré (phasmes et macumba); protecteur (père/mère des saints) et destructeur (Shango), subtil (mots subtils, mot dauphin) et émotif (colère d'étincelles).

21 Le dernier schème anaphorique est extrait du poème "Sentiments et ressentiments des mots » (23-24). Avec la répétition cinq fois de suite de la lexie « mot », Césaire assimile le mot aux animaux emblématiques des îles (touaou, strix, couresse). A ces réalités profanes font pièce les êtres fabuleux issus du panthéon amérindien (oiseau tonnerre: animaltotem sacré), européen (lémure : spectre d'un individu décédé dans la Rome antique) et extrême-oriental avec le dragon-du-lac.

C'est à un véritable travail sur le signifié et le signifiant que s'adonne Césaire. En ce qui concerne le signifié, nous pouvons noter des procédés comme la métaphorisation qui concourt à en faire une réalité concrète, palpable: "Fagots de mots qui dans les coins s'écroulent » (17); " mots lassos» (59-60); " pains de mots » (11-13). La personnification aussi : «patienter le mot » (22). Enfin la symbolisation : mot-macumba, mot shango (42), mot oiseau-tonnerre, (24). Dans ce dernier poème, ce sont les divinités africaines (shango, orisha de la foudre et du tonnerre chez les yorubas), amérindiennes (oiseau-tonnerre, animal totem sacré qui apporte la pluie), chinois (dragon-du-lac) et européen (lémure, spectre ou fantôme dans les croyances de la Rome antique). Ces entités des différents panthéons sont les symboles des peuples aux origines diverses qui, à force de brassages multiples, ont formé la population créole des Antilles d'aujourd'hui. Ce qui semble constant dans la construction de ces différentes images, c'est le recours au substantif comme épithète du mot (substantif adjectival). Tout se passe comme si le poète ne voulait pas se contenter de conférer un attribut au mot, mais plutôt l'assimiler aux termes imageants au point de les faire fusionner. Par ce procédé fort récurrent dans Moi, laminaire, Césaire opère non plus par qualification, mais plutôt par identification de l'objet-repère ou symbolisé (mot ici) aux divers termes imageants ou symbolisants.

En faisant voyager le mot d'une image à une autre, d'une isotopie à l'autre, ce dernier au terme de ses multiples avatars par lesquels il s'est assujetti d'autres valeurs finit par s'ériger en un "monstre sacré", une réalité terrible. Cette aventure est le lieu privilégié de toutes les unions possibles, des plus inattendues aux plus morganatiques.

Pour le travail sur le signifiant, deux principaux procédés seront retenus. D'abord l'anaphore que l'on retrouve dans deux poèmes: «mot-macumba " (deux anaphores successives) et "sentiments et ressentiments des mots ». Cette figure de répétition, en plus de produire un effet d'insistance de la lexie mot est bien, dans la plupart des cas, le lieu de l'éclosion de nombreuses images dont la profusion est amplifiée par l'accumulation de celles-ci.

Exemple : « le mot oiseau-tonnerre

le mot dragon-du-lac... » (23-24)

Puis l'homophonie entre mot et maux que Césaire exploite quelquefois.

Exemple : «Par quelques-uns des mots obsédant une torpeur

Et l'accueil et l'éveil de chacun de nos maux 
Je t'énonce FANON » (21)

Les mots sont le moyen d'expression des misères du poète et celles de son peuple. La parole se transmute en exutoire des douleurs individuelles et collectives.

Alors que «Mot-macumba» dévoile la fonctionnalité du mot pour le poète, dans « Internonce» (62), petit poème de trois strophes, Césaire insiste plutôt sur la relation qu'il entretient avec le mot. Il est «sa créature mais rebelle ». L'affection qu'il lui porte se révèle dans cette autre anaphore un petit mot, toute la charge affective étant supportée par l'adjectif petit ("Un petit mot saxifrage de tombeaux») (62). Le poète est celui qui actionne le mot (je te lance tiaulé). Il est sa créature dont il finit par perdre le contrôle pour devenir spectateur de son élan vertical (assistant à ton assaut sévère spectral et saccadé).

Le mot est réellement et constamment à l'aventure dans Moi, laminaire. Les procédés les plus récurrents dans ce jeu sur le signifiant et le signifié de la lexie mot sont principalement la métaphore, la personnification, la symbolisation, l'anaphore et l'homophonie.

En matière de poétique, il est certaines questions qui continuent de faire polémique, tant elles sont complexes et objet de multiples interprétations. L'application de l'aventure du mot au texte de Césaire nous servira de prétexte pour réexaminer quelques-unes d'entre elles, principalement la question du mot poétique et celle du statut du poète.

\section{Aventure du mot, mot poétique et du statut du poète}

Il s'agira pour l'essentiel de réévaluer les questions du mot poétique et du statut du poète à la lumière des acquis de la théorie de l'« aventure du mot ».

Le mot à l'aventure, pour Barthes, est un mot tout à la fois «libre, vertical, encyclopédique, monstre sacré» (Barthes, 1972: 39). Libre, parce qu'extrêmement réfractaire à toute contrainte, il refuse de s'assujettir à d'autres mots pour remplir la mission première de la langue: communiquer. En raison de sa liberté, il se dresse, vertical, rebelle à l'horizontalité de la chaine parlée. Ce qui en fait un mot « encyclopédique » que Barthes explique en ces termes:

Le mot est ici encyclopédique, il contient simultanément toutes les acceptions parmi lesquelles un discours relationnel lui aurait imposé de choisir. Il accomplit donc un état qui n'est possible que dans le dictionnaire ou dans la poésie, là où le nom peut vivre privé de son article, amené à une sorte d'état zéro, gros à la fois de toutes les spécifications passées et futures. (Ibid. : 39)

Enfin, le mot s'érige en monstre sacré, pour la simple raison qu'il engendre "un discours debout...plein de terreur. C'est-à-dire qu'il met l'homme en liaison non pas avec les autres hommes, mais avec les images les plus inhumaines de la Nature; le ciel. L'enfer, le sacré, l'enfance, la folie, la matière pure, etc. ».

31 Qu'est-ce qu'un mot poétique? Les avis sur la question sont divers et quelquefois divergents. Pour les uns (De Bonnefoy cité par Meschonnic, op.cit.: 58), le mot poétique nomme des essences contrairement aux autres mots qui «prennent trop clairement de l'extérieur l'acte humain, ne font que le décrire, n'ont pour contenu qu'un aspect ». Ce que l'on pourrait résumer par la formule suivante $:$ Nomen = noumen 
(mot $=$ chose en soi). Un tel mot relève de la parole comme verbe, parole créatrice, à l'instar du verbe johannique ou du Nommo, dans la mythologie dogon. ${ }^{4}$

D'autres ont voulu construire un langage poétique, une sorte de lexique propre à la poésie, un dictionnaire de la langue poétique. Les mots d'un tel dictionnaire auraient pour caractéristique fondamentale, comme le souligne Molinié, la rareté pour la raison toute simple qu'ils seraient difficiles à trouver hors poésie (exemple: cieux pour ciel; courroux pour colère; coursier pour cheval; hymen ; labeur....). Dessons (2005: 30) voit dans cette tentative improductive, un héritage du classicisme qui dénature plutôt la langue poétique par la caricature qu'elle en offre. In extenso, il pense qu'il ne saurait exister des éléments de la langue qui soient propres au discours poétique ou plus aptes à la poéticité : ni les mots, ni les personnes de la conjugaison, ni autres constituants de la langue. La conséquence pour l'analyse du poème est que celle-ci ne devrait pas accorder la primauté, a priori, à des éléments linguistiques particuliers, mais plutôt devrait embrasser toutes les unités du langage. L'analyse du poème est d'abord une analyse de discours. (Dessons, $2005: 31$ ).

En effet, dans la logique des rédacteurs de dictionnaire de la langue poétique, l'aventure du mot pourrait ne concerner que la catégorie de mots dits notionnels (doués de sens), à l'instar du nom, du verbe, de l'adjectif et des adverbes. Ainsi seraient à priori" inaptes" à l'aventure parce que vides de sens, les catégories de mots comme les conjonctions de coordination, les prépositions et tous les autres mots-outils. L'on serait alors contraint de distinguer dans la langue même des mots poétiques et non poétiques. Ce qui aurait pour conséquence d'inscrire la poéticité dans la nature ou l'essence des mots. Or, si la poésie est «fabrication", «création », c'est bien dans le choix et l'usage qu'un locuteur fait des mots que devrait résider la poéticité. Un mot ne naît pas poétique, il le devient par la virtuosité de l'usager de la langue, par ce qu'il est convenu d'appeler un processus de poétisation. Qui plus est, les mots de tous les jours peuvent devenir des mots poétiques. Des poètes comme Hugo, Mallarmé le revendiquent même. Le poète donne « un sens plus pur aux mots de la tribu » (Aquien et Molinié, Dictionnaire de rhétorique et de poétique: 594). Dans le glossaire de son œuvre Pour la poétique 1, Meschonnic propose la définition suivante du mot poétique :

Mot poétique. Degré valeur d'un mot qui n'apparait que dans l'œuvre. Tout mot peut être poétique. Exemple : araignée, puisque, quelqu'un chez Hugo ; botte chez Flaubert ; abeille, blanc, mais chez Apollinaire. (Meschonnic, 1970 : 174)

Il part du principe que tout mot peut être poétique et un même mot peut l'être diversement. Exit donc toute prétention à élaborer un dictionnaire de la langue poétique. La valeur d'un tel mot dépend tout à la fois de ses entours immédiats ("chaîne horizontale" ou co-texte) et lointains ("chaine verticale" ou contexte). En somme,

c'est un mot déformé-reformé : enlevé au langage puis travaillé, toujours le mot de la communication, en apparence, mais différent, d'une différence qui ne s'apprécie pas par un écart mesurable, mais par une lecture-écriture » (Meschonnic, 1970: 60-61).

Son repérage est facilité par la perception d'une anormalité ou incongruité (agrammaticalité dans la terminologie de Riffaterre) dans le texte ; le sens de base du mot jouant le rôle de «norme ».

L'on peut déduire de ce qui précède que le mot à l'aventure est un mot poétique. Plus il y en dans une œuvre plus celle-ci gagne en épaisseur littéraire. Toutefois, la poéticité ne dépend pas exclusivement de la quantité des mots à l'aventure, il faut aussi intégrer 
la qualité, autrement le degré d'aventure auquel le poète soumet les mots. Que la pression poétique s'exerce sur le signifiant ou sur le signifié, la finalité de celle-ci est d'en arriver à la rupture du mot avec son sens de base. In fine, ce qui demeure au terme de ce « marronnage » verbal, ce sont les valeurs, raison pour laquelle elles nous semblent premières.

37 Tous les mots dans un poème ne sont pas à l'aventure, fort heureusement, auquel cas le poème serait une suite de sons immotivés ou vides de sens. En dehors des comptines et autres jongleries verbales, la plupart des autres poèmes sont un savant mélange de mots employés dans leur sens de base et de mots à l'aventure. Ce qui facilite la tâche de l'analyste, qui peut alors les isoler pour les étudier. Si tous les mots devraient rompre les amarres avec leur sens de base, la frontière serait tenue entre la poésie et la musique, la première ferait figure d'antichambre de la seconde. Autrement, le summum de la poésie serait la musique (ce sont pourtant deux arts distincts quoique très proches, la poésie prise stricto sensu comme art du langage). Puisque les mots coupés de leur sens de base deviennent des sons, et le poème, une succession de sons, s'apparenterait à un morceau de musique. Loin s'en faut, d'abord parce que tous les mots dans un texte poétique ne sont pas à l'aventure et bien plus parce que l'aventure ne débouche jamais sur l'expiration du mot (rupture radicale entre son et sens), le lien de distend le plus loin possible, mais sans jamais rompre. Le corollaire d'une telle acception du mot est qu'elle érige le poète en "chef de bande». En effet, pour contraindre le mot à une telle verticalité, il faut un poète qui ne se contente plus d'orner le discours. Le poète doit avoir l'audace du corsaire et s'ériger en authentique " flibustier » de la langue, peu soucieux comme tout anticonformiste digne de ce nom, des règles séculairement établies, des convenances linguistiques ou sociales. Au bout de l'aventure, se découvre un Antigone, un Spartacus de la langue.

Le langage entre les mains de ce chef de bande que représente le poète, n'est plus le simple véhicule d'une pensée. Cette propriété originelle s'en trouve bouleversée. Les lexies peuvent suggérer une émotion, un état d'âme, l'indicible en somme. La langue n'est plus un simple véhicule, mais le lieu d'un jeu dont le maître reste incontestablement le poète entre les mains duquel le mot devient un matériau au service du beau, engagé qu'il est dans une sorte de tourbillon sans fin. C'est un mot terrible, tragique qui doit susciter effroi et terreur.

Le mot étant une réalité biface, sa mise en aventure consistera en un travail tant sur le signifié que le signifiant. Concernant le signifié, seront mis à profit les transferts de sens (métaphorisation, symbolisation, etc.). A ce niveau, les lexies s'échangent leurs valeurs ou s'assujettissent les valeurs des lexies environnantes. L'aventure implique aussi une exploitation artistique ou à des fins expressives du système connotatif, des relations sémantiques et des réseaux lexicaux entre autres. Cette aventure du mot pourrait concerner prioritairement le signifiant parce que la forme sonore du mot prime sur le contenu sémantique. Le rapprochement phonique peut permettre un apparentement des mots et générer de nombreuses figures de rhétorique comme les paronomases, les homéotéleutes. Au terme de l'aventure, les lexies totalement libérées de toutes contraintes s'affichent comme des signes debout, libres. C'est le triomphe de la verticalité sur l'horizontalité. Avec Césaire, il nous a été loisible d'apprécier la pression que son génie poétique a fait subir à la lexie mot, le matériau de base du poète et de tout artiste de la langue en général. La liberté, Césaire ne se contente pas de la rêver, de la réclamer à corps et à cris pour son peuple meurtri par des siècles de 
servitude ; il commence par l'expérimenter dans son écriture. Il libère alors le mot pour lui permettre d'investir des isotopies aussi éloignées les unes des autres, mais il explose aussi les carcans de l'horizontalité pour permettre une explosion de bouquets d'images, de symboles. Le poème devient le champ par excellence d'expérimentation de la liberté. En libérant le mot, le poète se libère lui-même et son peuple avec lui. L'étude des différents avatars du mot a permis de mettre au jour la relation particulière qu'il entretient avec celui-ci. Le mot, son matériau, lui est si intime qu'il en devient une réalité concrète. Il peut alors prendre la forme des différentes réalités de l'univers caribéen: faune et flore, mais aussi le minéral, les êtres et les phénomènes mythologiques originels (amérindiens) et ceux que l'histoire a transplanté sur cet archipel (africains, européens et indiens, chinois). L'aventure du mot comme méthode d'analyse des textes littéraires semble avoir été inspirée par la poésie moderne dont les caractéristiques fondamentales sont le déni de syntaxe et le sacre de la verticalité du mot. Le mot comme réalité libre et le statut du poète dont il a été question ci-dessus le montrent bien. Comme Meschonnic le souligne "autre poésie, autre "poétique" » (1970 : 17). Cette méthode d'analyse des textes déroge à cette règle car même inspirée par la poésie moderne, elle a vocation à s'appliquer à tout texte, à la seule condition que s'y trouvent des mots « debout ».

\section{BIBLIOGRAPHIE}

AQUIEN, Michèle et MOLINIÉ, Georges, Dictionnaire de rhétorique et de poétique, Librairie Générale Française, Paris, 1999.

BARTHES, Roland, Le degré zéro de l'écriture, suivi de Nouveaux essais critiques, Seuil, Paris, 1972.

BENVENISTE, Émile, Problèmes de linguistique générale 1, Gallimard, Paris, 1966.

CALAS, Fréderic, Leçons de stylistique, Armand Colin, Paris, 2011.

CÉSAIRE, Aimé, Moi, laminaire, Seuil, Paris, 1982.

CÉSAIRE, Aimé, « Poésie et connaissance », dans Aimé Césaire : l'homme et l'œuvre de Lilian

Kesteloot et Barthélemy Kotchy, Présence Africaine, Paris, 1973.

DESSONS, Gérard, Introduction à l'analyse du poème, Armand Colin, Paris, 2005.

DUCROT, Oswald et TODOROV, Tzevetan, Dictionnaire encyclopédique des sciences du langage, Seuil, Paris, 1972.

FROMILHAGUE, Catherine et SANCIER, Anne, Introduction à l'analyse stylistique, Bordas, Paris, 1991.

GARDES-TAMINE, Joëlle, La stylistique, Armand Colin, Paris, 2010.

JAKOBSON, Roman, Essai de linguistique générale, Éditions de Minuit, Paris, 1963.

MAZALEYRAT, Jean et MOLINIÉ, Géorges, Vocabulaire de la stylistique, PUF, Paris, 1989.

MESCHONNIC, Henri, Pour la poétique I, Gallimard, Paris, 1970.

MOLINIÉ, Géorges, Éléments de stylistique française, PUF, Paris, 1986. 
DE SAUSSURE, Ferdinand, Cours de linguistique générale (1916), Payot, Paris, 1969.

ZADI ZAOUROU, Bernard, « Aventure du mot et quête universaliste dans l'œuvre d'Aimé Césaire », dans CEuvres et Critiques, XIX, 2, Günter Narr Verlag, Tübingen, 1994.

ZADI ZAOUROU, Césaire entre deux cultures, NEA, Abidjan-Dakar, 1978.

\section{NOTES}

1. Voir Poésie et connaissance, extraits d'une communication donnée par Césaire au congrès de philosophie de Port-au-Prince (Haït) parue dans la revue Tropiques, 1944 et reprise par Lilyan Kestellot et Barthelemy Kotchy dans leur ouvrage Aimé Césaire, l'homme et l'œuvre (1973), Paris, Présence Africaine.

2. Degré zéro : on a pu le constater, si le premier degré relève de la dénotation, les trois autres niveaux ressortissent au champ des connotations. C'est à l'intérieur de ce champ que se situent les autres degrés d'expressivité.

3. Le marronnage désigne l'action pour un esclave noir de s'évader afin de vivre en liberté dans les bois. Il est considéré comme la forme première de la révolte contre l'esclavage, l'oppression des Noirs aux Antilles et dont la négritude est le prolongement.

4. Marcel Griaule en donne les détails dans son œuvre Dieu d'eau (1948).

\section{RÉSUMÉS}

Dans le prolongement des approches structuralistes, Bernard Zadi Zaourou, en prenant appui sur les travaux de Roland Barthes, Roman Jakobson et Aimé Césaire dans une certaine mesure, va élaborer une méthode d'analyse stylistique des textes littéraires : "l'aventure du mot ». Elle a pour objet l'analyse de tous les mots employés hors de leur sens de base. Il s'agit, à partir de l'analyse de ces mots, de souligner leur expressivité, avant de proposer du poème une hypothèse interprétative. Dans Moi, laminaire, il ne sera pas question de n'importe quelle lexie, mais du mot lui-même. Les récurrences multiples du « mot » dans divers poèmes du recueil d'Aimé Césaire le rendent particulièrement digne d'intérêt. L'application de cette méthode aux poèmes du chantre de la négritude offrira, par ailleurs, l'occasion d'un réexamen des questions du mot poétique et du statut du poète.

As a perpetuation of the structuralist approaches, Bernard Zadi Zaourou elaborates a method of stylistic analysis of literary texts: "The adventure of the word", based on works conducted by Roland Barthes, Roman Jakobson and Aimé Césaire. It deals with the analysis of all the words that are used regardless of their basic meaning. From the analysis of these words, it is all about underlining their expressiveness, before proposing an interpretative hypothesis of the poem. In Moi, laminaire, it is not about any lexical feature, but about the word itself. Its multiple recurrences in diverse poems of Aimé Césaire's collection, make it particularly worthy of interest. The application of this method to the poems of the cantor of negritude will also offer an opportunity for a reexamination of the issue of the poetic word as well as of the status of the poet. 
INDEX

Mots-clés : lexie, aventure du mot, rendement stylistique, signifiance, mot poétique

$$
\text { لكسيم, مغامرة كلمة, المردودية الأسلوبية, دلالة, كلمة شعريةفهرس الكلمات المفتاحية: }
$$

Keywords : lexical Feature, adventure of the word, stylistic output, significance, poetic word

\section{AUTEUR}

\section{SYLVANIUS BOBO ROSTAND}

Université Félix Houphouët-Boigny Abidjan 